\title{
Propagation of Density Fluctuations in Nonuniform Fluids: Simple Models
}

\author{
James C. Rainwater* \\ National Bureau of Standards, Boulder, CO 80303
}

May 4, 1981

\begin{abstract}
As a first step toward the understanding of Rayleigh-Brillouin scattering from a fluid with a temperature gradient, we analyze the initial value problem for certain prototype one-dimensional nonuniform systems. For sufficiently short times and localized initial pulses, it is not necessary to impose actual physical boundaries on the linearly nonuniform system. By a straightforward, though unconventional, application of Fourier and Laplace transform methods, explicit and physically reasonable solutions are constructed for the propagation of fluctuation pulses in space and time according to the nonuniform wave and diffusion equations and a special case of the nonuniform damped wave equation. The analog of the dynamic structure factor is also constructed for the latter two cases.
\end{abstract}

Key words: damped wave motion; density fluctuations; diffusion; dynamic structure factor; initial value problem; nonuniform systems; one-dimensonal models; Rayleigh-Brillouin scattering.

\section{Introduction}

There has been considerable recent interest in the problem of Rayleigh-Brillouin scattering in a fluid with a stationary temperature gradient. [1-10] ${ }^{1}$ The problem, in essence, is to generalize Mountain's standard derivation of the equilibrium Rayleigh-Brillouin spectrum [11] to the nonequilibrium stationary state.

Laser light is scattered from a liquid because of random density (and therefore dielectric constant) fluctuations. $[12,13]$ In the hydrodynamic regime, a density fluctuation will propagate in time partly as a diffusion or "heat" mode, causing the Rayleigh line, and partly as a wave or "sound" mode, causing the two Brillouin lines. With a temperature gradient the spectrum is altered. For example, if more sound modes are generated at the hot side of the liquid than at the cold side, there will be a larger flux of sound modes from hot-to-cold than from cold-to-hot, thereby causing a size asymmetry in the Brillouin peaks. Some treatments of this problem, [1-4] in fact, predict no spectral changes other than an overall asymmetric scale factor in the Brillouin peaks. Such an asymmetry has recently been observed experimentally. [14]

Other authors [5-9] predict changes in the mathematical shape of the Brillouin lines from their equilibrium (Lorentzian) form. Such changes of shape, very difficult to measure experimentally, are interpreted here as the effect of propagation of modes through regions of changing sound speed, sound attenuation, and thermal diffusivity. Among the theories published to date, [1-10] there is significant disagreement as to the precise form of the nonequilibrium spectrum. Some discrepancies may arise from different assumptions about the nature of the nonequilibrium steady state, but it is not clear at this time if all do.

None of the theories published thus far appear to follow the procedure of ref. 11 explicitly, i.e., to solve the hydrodynamic equations for the time evolution of a density fluctuation and, subsequently, to perform a 
stochastic and ensemble average to determine the dynamic structure factor and hence the spectrum of scattered light. Closest in spirit to such a procedure is the hydrodynamic approach of van der Zwan and Mazur, $[7,8]$ but they appear to bypass the calculation of the time development of an individual fluctuation.

An explicit calculation of the evolution in time and (both physical and Fourier) space of a spontaneous density fluctuation, in the presence of a temperature gradient, should shed considerable light on the presently confused predictions of Rayleigh-Brillouin scattering. The possibility of changes with temperature, and hence distance, of all physically relevant variables should be considered. Significantly, possible changes in the shape of the Rayleigh line have been substantially neglected thus far. [1-10]

This paper represents an initial step in the execution of the above program. Since the full threedimensional problem of coupled equations in density and temperature fluctuations is very complex mathematically, it is illuminating to deal first with simplified models. Here we solve explicitly the onedimensional wave, diffusion, and damped wave equations to first order in nonuniformity. Care is taken to select nonuniform equations which correspond as closely as possible to the physical nonequilibrium steadystate problem.

Our technique is a generalization of a method due to Brownell [15] to calculate the Green's function for a nonuniform wave equation. We show that the initial value problem for the nonuniform wave, diffusion, and damped wave equations can indeed be solved, to linear order in nonuniformity, in a manner which closely parallels Mountain's derivation. The methods are systematic, but the mathematics becomes rapidly more difficult with greater complexity of the underlying differential equations. Our eventual goal, yet to be attained, is the explicit solution of the coupled hydrodynamic equations and determination of the dynamic structure factor in one and, if possible, three dimensions. Since wave and diffusion equations are prevalent throughout physics, the techniques presented here may prove useful for a variety of other physical problems as well.

We begin in section 2 with a general discussion of some predictable mathematical difficulties. Section 2.1 examines the applicability of the initial value problem to Rayleigh-Brillouin scattering in a fluid with a temperature gradient. Section 2.2 describes the needed transformations of variable and examines the range of validity of a first-order nonuniformity calculation. Solutions to the various initial value problems in the uniform limit, mostly well-known, are outlined in Section 3 . The subsequent three sections present explicit solutions, with intuitively oriented interpretations, to the initial value problem for the nonuniform wave, diffusion, and damped wave equations respectively. Results are summarized in Section 7.

\section{Initial mathematical considerations}

\subsection{The initial value problem}

The basic relationship between the measured spectrum of scattered light and the structure of a liquid sample is $[12,13]$

$$
I(\omega)=C S(\mathbf{k}, \omega)
$$

where $\omega$ is the shift in frequency and $\mathbf{k}$ the shift in wave vector of the scattered light, $I$ is the average measured intensity, and $C$ is a constant, not important for our present interests. $S(\mathbf{k}, \omega)$, the dynamic structure factor, is the Fourier transform in time of the intermediate scattering function $F(\mathbf{k}, t)$

$$
S(\mathbf{k}, \omega)=\int_{-\infty}^{\infty} e^{-i \omega t} F(\mathbf{k}, t) d t
$$

where

$$
F(\mathbf{k}, t)=\langle\bar{n}(-\mathbf{k}, 0) \bar{n}(\mathbf{k}, t)\rangle .
$$


Here the brackets denote an ensemble average and $\bar{n}(\mathbf{k}, t)$ is the Fourier transform in space of $\mathbf{n}(\mathbf{r}, t)$, the fluctuation of liquid density about equilibrium, i.e.,

$$
\bar{n}(\mathbf{k}, t)=\int d \mathbf{r} e^{-i \mathbf{k} \cdot r} n(\mathbf{r}, t) .
$$

The hydrodynamic regime is defined by the inequality

$$
k^{-1}>>\ell
$$

where $\ell$ is the interparticle spacing, and

$$
k=2 k_{i} \sin \frac{\theta}{2},
$$

where $k_{i}$ is the wave vector of the incident light and $\theta$ is the angle by which the light is scattered. In a liquid $\ell$ is small and scattering experiments with visible light are always in the hydrodynamic regime. For dilute gases $\ell$ is large, but the hydrodynamic regime can be attained [16] with sufficiently small $\theta$. Kirkpatrick, et al. [5] consider the nonuniform problem starting from the Boltzmann equation, which is strictly valid only for dilute gases. The initial experimental observation of changes in the scattered spectrum due to a temperature gradient has been made with liquid water at a very low scattering angle. [14].

Mountain's derivation [11] of Rayleigh-Brillouin scattering in equilibrium begins with the observation of Landau and Placzek [17] that, in the hydrodynamic regime, density fluctuations, $n(\mathbf{r}, t)$, and temperature fluctuations, $T(\mathbf{r}, t)$, satisfy a set of coupled second-order linear differential equations. After a Fourier transformation in space and a Laplace transformation in time, those equations become coupled algebraic equations which involve the initial $(t=0)$ values and time derivatives of the fluctuations. The derivation of ref. [1]], in effect, makes the further simplifications

$$
\begin{aligned}
& \stackrel{\bullet}{n}(\mathbf{k}, 0)=0 \\
& \bar{T}(\mathbf{k}, 0)=0
\end{aligned}
$$

where a dot denotes a time derivative. These simplifications are not literally correct, but are permissible because, at the end of the calculation when the autocorrelation function is evaluated, the above quantities are not correlated with the initial density fluctuations. In particular, because of time reversal invariance,

$$
<\bar{n}(-\mathbf{k}, 0) \dot{\bullet}(\mathbf{k}, 0)>=0
$$

and because of the thermodynamic independence of density and temperature,

$$
<\bar{n}(-\mathbf{k}, 0) \bar{T}(\mathbf{k}, 0)>=0 .
$$

The solution of the differential equations leads to a dynamic structure factor, eq. (2), in terms of the static structure factor

$$
S(k)=\langle\bar{n}(-\mathbf{k}, 0) \bar{n}(\mathbf{k}, 0)>
$$

which must be determined from separate (but well-known) thermodynamic considerations. [12, 18] An alternate method to the above initial value problem is to consider the response of the system to a random fluctuating force. [19]

In the presence of a stationary temperature gradient, time-reversal symmetry no longer holds, $[1,4]$ and eq (9) is no longer satisfied. Thus an initial value calculation, by itself, does not suffice to determine the altered Rayleigh-Brillouin spectrum. However, an initial value approach may suffice to determine "shape effects," as opposed to "size effects," in the altered spectrum. 
More precisely, we define $\epsilon$, which is proportional to $|\nabla \mathrm{T}|$, as the appropriate dimensionless expansion parameter. The LHS of eq (9) will be $O(\epsilon)$ due to differences in the rate of generation of fluctuations at different positions in the fluid. These differences will lead to overall asymmetries in the Brillouin lines. A quite different effect is the change in the shape of the Rayleigh and Brillouin lines due to propagation of the unperturbed fluctuations through a nonuniform medium.

In the linearization of the problem about uniformity, it is reasonable to assume that the two effects are independent and additive. Further corrections due to the nonuniform propagation of fluctuations which are themselves generated by nonuniformity presumably lead to second order, $O\left(\epsilon^{2}\right)$ corrections.

Thus an initial value problem approach to Rayleigh-Brillouin scattering in a temperature gradient, while incomplete, may suffice to determine the shape changes of the lines, and hence will complement those approaches which appear to emphasize only size changes. [1, 4]

\subsection{Transformations and nonuniformity expansions}

In the presence of a temperature gradient, any relevant physical property $A$ of a fluid may be expanded in a Taylor series about some convenient origin of position $(\mathbf{r}=0)$ in the fluid, i.e.,

$$
A(\mathbf{r})=A(0)+(\mathbf{r} \cdot \nabla T)\left(\frac{\partial A}{\partial T}\right)_{r=0}+\ldots
$$

For the linearized problem, only the above two terms are retained.

The coefficients of the hydrodynamic equations, in equilibrium eqs. (6)-(8) of ref. [11], will then be position-dependent according to eq (12). Since the differential operators do not commute with $\mathbf{r}$, it is important to return to the fundamental derivations of the differential equations governing the processes, to determine in what order differential operators and position-dependent coefficients should be placed.

In the prototype one-dimensional equations considered here, the coefficients will have the position dependence

$$
a(x)=a_{0}+a_{1} x
$$

where $a_{1}$ is assumed to be small.

We will examine for the various differential equations the initial value problem, i.e., the solution for $y(x, t)$ given $y(x, 0)$ and zero initial time derivative,

$$
\dot{y}(x, 0)=0 .
$$

Equation (14) is not necessary for the diffusion equation, which is first order in time.

It is convenient at the outset to introduce a special notation for the several variable spaces which are required. Physical space or "P-space" denotes the variables $\{x, t\}$. Fourier space or "F-space" consists of the variables $\{k, t\}$, where

$$
\bar{y}(k, t)=\int_{-\infty}^{\infty} d x e^{-i k x} y(x, t) .
$$

Laplace space or " $L$-space" is formed by the variables $\{x, s\}$, where

$$
\hat{y}(x, s)=\int_{-\infty}^{\infty} d t e^{-s t} y(x, t) .
$$

Finally, Fourier-Laplace space or "FL-space" consists of the variables $\{k, s\}$, where

$$
\tilde{y}(k, s)=\int_{-\infty}^{\infty} d x e^{-i k x} \hat{y}(x, s)=\int_{-\infty}^{\infty} d t e^{-s t} \bar{y}(k, t) .
$$

Our convention for transformed functions is that a bar denotes $F$-space, a caret $L$-space, and a tilde $F L$-space. 
The differential equations considered here require that certain coefficients be positive, which is, in general, in conflict with eq (13). For small but finite $a_{1}$, if

$$
x<-a_{0} / a_{1}
$$

then $a(x)<0$, which could lead to physical nonsense, e.g., negative diffusion or imaginary wave speed. We must assume that such large negative values of position are irrelevant to the problem.

A possible escape from this difficulty is to impose explicit boundaries on the linearly nonuniform region of space with, for example, separate uniform regions outside those boundaries.

$$
\begin{aligned}
a(x) & =a^{(1)} & & x \leq-L \\
& =\frac{1}{2}\left[a^{(1)}+a^{(2)}\right] & & \\
& +\frac{1}{2}\left[a^{(2)}-a^{(1)}\right] \frac{x}{L} & & -L \leq x \leq L \\
& =a^{(2)} & & x \geq L
\end{aligned}
$$

We elect, however, not to proceed in this manner because a new parameter $L$ is introduced into the problem, and the exact solution for a general $L$ is likely to be mathematically cumbersome. Furthermore, for the conditions we seek to impose, there should exist a unique linearized solution

$$
y(x, t)=y^{(0)}(x, t)+a_{1} y^{(1)}(x, t)+O\left(\epsilon^{2}\right),
$$

where $y^{(0)}$ is the uniform solution, which is independent of $L$. These conditions are that the initial value packet be localized and that the time be small compared to the time required for the packet to propagate (in significant measure) to the region of negative $a$. We consider initial value packets of finite width $w_{o}$, i.e.,

$$
y(x, 0)=0 \quad|x|>\frac{w_{0}}{2}
$$

and the "smallness" of $a_{1}$ is made precise in dimensionless form by

$$
\frac{a_{1} w_{0}}{a_{0}}<<1
$$

Additionally, we require a constraint on the time $\ell$ of propagation. For example, the solution to the uniform wave equation moves with constant velocity $c$, so we require times short enough that

$$
\frac{a_{1} c t}{a_{0}}<<1
$$

or, for the diffusion equation, since the mean-square width of an initial delta-function pulse is (in one dimension)

$$
\left\langle x^{2}\right\rangle=2 D t
$$

the appropriate condition on the time is

$$
a_{1}(2 D \ell)^{1 / 2} / a_{0}<<1 .
$$

Calculation of the dynamic structure factor, in principle, requires an integral from $t=0$ to $t=\infty$. Nevertheless, we expect the linear nonuniformity theory to give a meaningful structure factor if the fluctuation has substantially decayed away in a time such that the inequalities (21) and (23) are valid. The phrase "decay away" is imprecise; a norm of the fluctuation needs to be defined and must be shown to be negligible after some particular time interval. 
One possible norm is

$$
\|y\|=\int_{-\infty}^{\infty} d x y(x, t)
$$

but, for the equations considered here, eq (24) happens to be a constant of the motion, and does not decay in time at all.

What do decay in time however, are Fourier components of the fluctuation for finite $k$. The $k=0$ componenet is $\|y\|$ itself. For diffusion, $\bar{y}(k, t)$ decays as $\mathrm{e}^{-D k^{2}}$, and for the damped wave equation considered here, it decays as $\mathrm{e}^{-\Gamma \boldsymbol{k}^{2} t}$, where $\Gamma$ is the attenuation coefficient. In the Rayleigh-Brillouin experiment, a particular value of $k$ is monitored by virtue of the fixed laser light frequency and scattering angle. If we are similarly interested in monitoring a particular value $k=k_{\text {o }}$, then for the times

$$
t_{D}=\left(D k_{\mathrm{o}}^{2}\right)^{-1}
$$

and

$$
t_{\Gamma}=\left(\Gamma k_{o}^{2}\right)^{-1}
$$

we require that inequalities (21) and (23) hold. The various inequalities for our particular applications in terms of variables defined in later sections are listed in table $\mathrm{I}$.

TAble 1.

\begin{tabular}{lll}
\hline $\begin{array}{c}\text { Differential } \\
\text { Equation }\end{array}$ & \multicolumn{1}{c}{$\begin{array}{c}\text { Conditions for } \\
\text { Validity of } \\
y(x, t)\end{array}$} & $\begin{array}{c}\text { Conditions for } \\
\text { Validity of } \\
S(k, \omega)\end{array}$ \\
\hline Undamped wave & $\frac{b w_{0}}{a}<<1$ & Never valid \\
& $\frac{b c t}{a}<<1$ & \\
Diffusion & $\frac{b w_{0}}{a}<<1$ & $\frac{b}{a k}<<1$ \\
Damped wave & $\frac{b}{a}\left(D_{0} t\right)^{1 / 2}<<1$ & \\
& $\frac{b w_{0}}{a}<<1$ & \\
$\left(\frac{\Gamma k}{c}<<1\right)$ & $\frac{b c t}{a}<<1$ & $\frac{b c}{a \Gamma k^{2}}<<1$ \\
\hline
\end{tabular}

The standard method of solution to the initial value problem for a partial differential equation in $P$-space is to transform to $F L$-space. In the uniform cases, the equation is an algebraic one and is easily solved. The solution is completed upon inverse transformation to $P$-space. In ref. [11] only the inverse transformation to $F$-space is required to obtain the intermediate scattering function, eq (3), and the dynamic structure factor, eq (2). 
Since, under Fourier transformation

$$
x \rightarrow i \frac{\partial}{\partial k}
$$

a partial differential equation in P-space with linearly nonuniform coefficients becomes, in $F L$-space, a linear differential equation in $k$. It is always possible to solve a linear differential equation by the integrating factor technique. [20] However, a unique solution requires a boundary value on $k$, and the problem appears at first not to specify any particular boundary value. We shall see that this apparent new degree of freedom is bogus and that the physics imposes a particular boundary value on $k$.

A more cumbersome problem is the inverse transformation. As stated previously, in $P$-space the solution is nonzero only within a width $w$ about the origin such that $(20)$ holds. In $F$-space, according to Heisenberg's inequality, $[21]$ the solution $\bar{y}(k, t)$ has a width $w_{o}^{-1}$. Only the values of $x$ such that

$$
\frac{a_{1}|x|}{a_{o}}<<1
$$

are significant; however, in $F$-space there is no analogous inequality. For example,

$$
\frac{a_{1}}{a_{0}|k|}<<1
$$

does not hold for all significant values of $|k|$, since such values of $|k|$ can range from zero to $w_{0}^{-1}$.

We conclude that there is no natural means, in $F L$-space or $F$-space, to take advantage of the smallness of the nonuniformity parameter. Therefore, after solving the linear differential equation in $F L$-space, we must first inverse transform to $L$-space (and expand in nonuniformity where convenient), then inverse transform to $P$-space (and further expand in nonuniformity), and lastly transform to $F$-space. This circuitous but required route is shown schematically in figure 1.

\section{Expand in nonuniformity}

\section{P space}

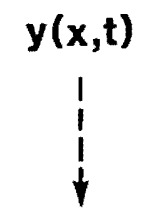

F space



L space

$\hat{\mathbf{y}}(\mathbf{x}, \mathbf{s})$

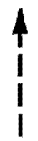

FL space

$$
\tilde{\mathbf{y}}(\mathbf{k}, \mathbf{s})
$$

$$
\mathbf{S}(\mathbf{k}, \omega)
$$

Figure 1. Sequence of transformations for the uniform problem (solid arrows) and for the nonuniform problem (dashed arrows).

In the following sections we carry out the above procedure explicitly for three nonuniform differential equations, after first reviewing their respective uniform solutions. 


\section{Uniform differential equations}

In this section, for reference purposes, we briefly outline the soluions to the initial value problem for the one-dimensional uniform wave, diffusion, and damped wave equations. These results are well-known.

\subsection{Wave equation}

The wave equation in $P$-space is

$$
\frac{\partial^{2} y}{\partial x^{2}}-\frac{1}{c^{2}} \frac{\partial^{2} y}{\partial t^{2}}=0
$$

We assume a given initial wave packet $y(x, 0)$ with zero initial time derivative. The equation is $F L$-space is

$$
-k^{2} \tilde{y}(k, s)-\frac{s^{2}}{c^{2}} \bar{y}(k, s)+\frac{s}{c^{2}} \bar{y}(k, 0)=0
$$

with the solution

$$
\bar{y}(k, s)=\frac{s \bar{y}(k, 0)}{\mathrm{s}^{2}+c^{2} k^{2}} .
$$

The solution in $F$-space is

$$
\bar{y}(k, t)=\frac{1}{2} \bar{y}(k, 0)\left[e^{i c k t}+e^{-i c k t}\right],
$$

the solution in $L$-space is

$$
\hat{y}(x, s)=\frac{1}{2 c} \int_{-\infty}^{\infty} d u e^{-y|u| / c} y(x-u, 0)
$$

and the solution in $P$-space is d'Alembert's solution,

$$
y(x, t)=\frac{1}{2}[y(x-c t, 0)+y(x+c t, 0)],
$$

i.e., the pulse divides in half and each half moves, without change of shape, at speed $c$. For an ensemble of packets, the analog of the intermediate scattering function is

$$
<\bar{y}(-k, 0) \bar{y}(k, t)>=\frac{1}{2}<\bar{y}(-k, 0) \bar{y}(k, 0)>\left[e^{i c k t}+e^{-i c k t}\right]
$$

and its Fourier transform in time, the analog of the dynamic structure factor, is

$$
S(k, \omega)=\pi<\bar{y}(-k, 0) \bar{y}(k, 0)>[\delta(\omega-c k)+\delta(\omega+c k)]
$$

i.e., two "Brillouin lines" of infinitely small width, since there is no damping.

\subsection{Diffusion equation}

The diffusion equation in $P$-space is

$$
\frac{\partial y}{\partial t}-D \frac{\partial^{2} y}{\partial x^{2}}=0 .
$$


An initial wave packet $y(x, 0)$ is given. Since the equation is first-order in time, no initial time derivative is specified. The equation in $F L$-space is

$$
s \tilde{y}(k, s)-\bar{y}(k, 0)+D k^{2} \tilde{y}(k, s)=0
$$

with solution

The solution in $F$-space is

$$
y(k, s)=\frac{\bar{y}(k, 0)}{s+D k^{2}}
$$

$$
\bar{y}(k, t)=\bar{y}(k, 0) e^{-D x^{2} t}
$$

the solution in $L$-space is

$$
\hat{y}(x, s)=\frac{1}{2}(s D)^{-1 / 2} \int_{\infty}^{\infty} d v y(v, 0) e^{-(s / D)^{1 / 2}|x-v|,}
$$

and the solution in $P$-space is

$$
y(x, t)=\frac{1}{2(\pi D t)^{1 / 2}} \int_{-\infty}^{\infty} d v y(v, 0) \mathrm{e}^{-(x-v)^{2 / 4 D t}} .
$$

For a $\delta$-function initial condition, $y(x, 0)=\delta(x)$, the solution takes the familiar form

$$
y(x, t)=\frac{1}{2(\pi D t)^{1 / 2}} e^{-x^{2} / 4 D_{t}} .
$$

The analog of the intermediate scattering function is

$$
<\bar{y}(-k, 0) \bar{y}(k, t)>=\left\langle\bar{y}(-k, 0) \bar{y}(k, 0)>e^{-D k^{2} t}\right.
$$

and the analog of the dynamic structure factor is

$$
S(k, \omega)=<\bar{y}(-k, 0) \bar{y}(k, 0)>\frac{2 D k^{2}}{\omega^{2}+\left(D k^{2}\right)^{2}},
$$

i.e., a Lorentzian "Rayleigh line" with width $D k^{2}$.

\subsection{Damped wave equation}

There are several possible choices for a wave equation with damping. We choose the case that corresponds most closely to the Rayleigh-Brillouin problem, i.e., an equation with " $k^{2}$-damping." In $P$-space this is

$$
\frac{\partial^{2} y}{\partial x^{2}}+\frac{2 \Gamma}{c^{2}} \frac{\partial^{2}}{\partial x^{2}} \frac{\partial y}{\partial t}-\frac{1}{c^{2}} \frac{\partial^{2} y}{\partial t^{2}}=0 .
$$

We again begin with an initial wave packet $y(x, 0)$ with zero initial time derivative. The equation in $F$-space is

$$
k^{2} \bar{y}(k, t)+\frac{2 \Gamma k^{2}}{c^{2}} \frac{\partial}{\partial t} \bar{y}(k, t)+\frac{1}{c^{2}} \frac{\partial^{2}}{\partial t^{2}} \bar{y}(k, t)=0
$$

which is the equation for a damped harmonic oscillator. The condition that the oscillation be underdamped, which we impose here, is

$$
\frac{\Gamma k}{c}<1
$$


The equation in $F L$-space is

$$
\begin{gathered}
k^{2} \bar{y}(k, s)+\frac{2 \Gamma s k^{2}}{c^{2}} \bar{y}(k, s)-\frac{2 \Gamma k^{2}}{c^{2}} \bar{y}(k, 0)+\frac{s^{2}}{c^{2}} \tilde{y}(k, s) \\
-\frac{s}{c^{2}} \bar{y}(k, 0)=0
\end{gathered}
$$

with the solution

$$
\tilde{y}(k, s)=\bar{y}(k, 0) \frac{s+2 \Gamma k^{2}}{s^{2}+2 \Gamma s k^{2}+c^{2} k^{2}}
$$

The solution in $F$-space is

$$
\begin{gathered}
\bar{y}(k, t)=\frac{1}{2} \bar{y}(k, 0)\left\{e^{-\Gamma k^{2} t}\left[e^{\left.i\left(c^{2}-\Gamma^{2} k^{2}\right)\right)^{1 / 2 k t}}+e^{-i\left(c^{2}-\Gamma^{2} k^{2}\right)^{1 / 2 k t}}\right]\right. \\
\left.+\frac{\Gamma k}{i\left(c^{2}-\Gamma^{2} k^{2}\right)^{1 / 2}} \mathrm{e}^{-\Gamma k^{2} t}\left[e^{i\left(c^{2}-\Gamma^{2} k^{2}\right)^{1 / 2 k t}}-e^{-i\left(c^{2}-\Gamma^{2} k^{2}\right)^{1 / 2 k t}}\right]\right\}
\end{gathered}
$$

and the solution in $L$-space is

$$
\begin{gathered}
\hat{y}(x, s)=\frac{1}{2\left(c^{2}+2 \Gamma s\right)^{1 / 2}} \int_{-\infty}^{\infty} d v\left[v(v, 0)-\frac{2 \Gamma}{s} \frac{\partial^{2}}{\partial v^{2}} y(v, 0)\right] \\
\times e^{-s|x-v| /\left(c^{2}+2 \Gamma s\right)^{1 / 2}} .
\end{gathered}
$$

However, to the best of our knowledge, there is no representation of the solution in $P$-space in terms of elementary functions. But a convenient approximate solution in $P$-space may be obtained by means of a dispersion expansion. First, the concept of a $k$-dependent wave speed is introduced, i.e.,

$$
c(k)=c\left(1-\frac{\Gamma^{2} k^{2}}{c^{2}}\right)^{1 / 2}
$$

If $\frac{\Gamma k}{c}<<1$ (extreme underdamping) we may expand in this small "dispersion parameter." Then eq (44), to leading order, is

$$
\bar{y}(k, t)=\frac{1}{2} \bar{y}(k, 0)\left\{e^{-r k^{2} t}\left[e^{i c k t}+e^{-i c k t}\right]\right\}
$$

with a corresponding solution in $P$-space

$$
y(x, t)=\frac{1}{4(\pi \Gamma t)^{1 / 2}} \int_{-\infty}^{\infty} d v y(v, 0)\left[e^{-(x-v+c t)^{2} / 4 \Gamma t}+e^{-(x-v-c t)^{2} / 4 \Gamma t}\right]
$$

which corresponds to two wave pulses travelling in opposite directions and spreading with time in a diffusionlike manner. In the nonuniform case there will be expansions in both dispersion and nonuniformity; however it is reasonable to assume that the two expansions are independent of each other.

From the lowest-order solution, eq (54), the analog of the intermediate scattering function is

$$
<\bar{y}(-k, 0) \bar{y}(k, t)>=\frac{1}{2}<\bar{y}(-k, 0) \bar{y}(k, 0)>e^{-\Gamma k^{2} t}\left[e^{i c k t}+e^{-i c k t}\right]
$$

and the analog of the dynamic structure factor is

$$
S(k, \omega)=\left\langle\bar{y}(-k, 0) \bar{y}(k, 0)>\times\left[\frac{\Gamma k^{2}}{(\omega-c k)^{2}+\left(\Gamma k^{2}\right)^{2}}+\frac{\Gamma k^{2}}{(\omega+c k)^{2}+\left(\Gamma k^{2}\right)^{2}}\right],\right.
$$

i.e., two Lorentzian "Brillouin lines" each with width $\Gamma k^{2}$. 


\section{Nonuniform wave equation}

Having reviewed the initial value problem for the three uniform differential equations, we next derive solutions to their nonuniform counterparts. The solutions must have the form of the uniform solution plus a small correction linear in nonuniformity. In all cases the nonuniformity parameter will be denoted by the variable $b$. The solutions are subject to the constraints on time, distance, and wavenumber described in section 2.2 .

In the following three sections we first present the exact form of the nonuniform differential equation which we take to be (in one dimension) closely analogous to the three-dimensional Rayleigh-Brillouin experimental situation described earlier. Thereafter we present a detailed mathematical derivation of the solution. Each selection then ends with a physical interpretation of the solution thus derived.

\subsection{The differential equation}

Following Brownell, [15] we write the nonuniform wave equation as

$$
\varrho(x) \frac{\partial^{2} y}{\partial t^{2}}-T \frac{\partial^{2} y}{\partial x^{2}}=0
$$

where

$$
\varrho(x)=a+b x
$$

Physically, this corresponds to a stretched string with a variable mass per unit length $\varrho(x)$ at a constant tension $T$. This is analogous to the fluid with a stationary temperature gradient, where the pressure is constant throughout but the density varies (to first order linearly) with distance due to the gradient in temperature. Again we consider a given initial pulse $y(x, 0)$ with zero initial time derivative.

\subsection{Solution}

The equation in $F L$-space is

$$
\begin{gathered}
\frac{\partial}{\partial k} \hat{y}(k, s)-\left[\frac{i a}{b}+\frac{T k^{2} i}{b s^{2}}\right] \hat{y}(k, s)= \\
\frac{1}{\mathrm{~s}} \frac{\partial}{\partial k} \bar{y}(k, 0)-\frac{a i}{b s} \bar{y}(k, 0) .
\end{gathered}
$$

The first-order differential equation in $k$ is solved by standard methods [20] as

$$
\begin{aligned}
y(k, s) & =\int_{k}^{k_{1}} d k^{\prime}\left[\frac{a i}{b s} \bar{y}\left(k^{\prime}, 0\right)-\frac{1}{s} \frac{\partial}{\partial k^{\prime}} \bar{y}\left(k^{\prime}, 0\right)\right] \\
& \times \exp \left[-\frac{i a\left(k^{\prime}-k\right)}{b}-\frac{T i\left(k^{\prime 3}-\mathrm{k}^{3}\right)}{3 b s^{2}}\right] .
\end{aligned}
$$

Formally, the integration constant $k_{1}$ appears to give a new and unwanted degree of freedom to the problem. However, as pointed out by Brownell [15], the existence of an inverse transformation requires certain analyticity properties in the complex $s$-plane. In our formulation, the requirement is that $\mathfrak{y}(k, s)$ be analytic in the right half of the complex $s$-plane, which in turn requires that $\left(k^{\prime 3}-k^{3}\right)$ be the same sign for all $k$. This requires that $k_{1}=\infty$. We also henceforth assume, without loss of generality, that $b$ is positive.

Changing variables to $u=k^{\prime}-k$ yields

$$
\begin{gathered}
\hat{y}(k, s)=\int_{b}^{\infty} d u\left[\frac{a i}{b s} \bar{y}(u+k, 0)-\frac{1}{s} \frac{\partial}{\partial k} \bar{y}(u+k, 0)\right] \\
\times \exp \left[-\frac{i a u}{b}-\frac{T i}{3 b s^{2}} u\left(u^{2}+3 k u+3 k^{2}\right)\right] .
\end{gathered}
$$


As explained in section 2.2 , we must now transform to $L$-space. Let $\mathcal{F}^{-1}$ denote the inverse Fourier transform operator, i.e.,

$$
\Im^{-1}\{f(k)\}=\frac{1}{2 \pi} \int_{-\infty}^{\infty} d k e^{i k x} f(k)
$$

Now,

$$
\begin{gathered}
\mathcal{P}^{-1}\left[\frac{a i}{b s} \bar{y}(u+k, 0)-\frac{1}{s} \frac{\partial}{\partial k} \bar{y}(u+k, 0)\right] \\
=\frac{a i}{b s} y(x, 0) e^{-i \omega x}\left(1+\frac{b x}{a}\right) .
\end{gathered}
$$

Next we require the inverse Fourier transform of the second term in eq (62). The relation

$$
\int_{-\infty}^{\infty} d k e^{-\alpha k^{2}-\beta k}=\left(\frac{\pi}{\alpha}\right)^{1 / 2} e^{\beta^{2} / 4 \alpha}
$$

holds for complex $\alpha$ and $\beta$ providing $R e \alpha>0$. To apply eq (65), we must assign a small negative imaginary component to $T$, i.e.,

$$
T \rightarrow T-i \epsilon
$$

with the limit $\epsilon \rightarrow 0$ taken at the end of the calculation. It then follows that

$$
\begin{gathered}
\mathcal{P}^{-1}\left\{\exp \left[-\frac{i a u}{b}-\frac{T i}{3 b s^{2}} u\left(u^{2}+3 k u+3 k^{2}\right)\right]\right\} \\
=\frac{1}{2} s(b / \pi u i T)^{1 / 2} \exp \left[\frac{i}{4}\left(\frac{T u^{3}}{b s^{2}}-2 u x+\frac{b s^{2} x^{2}}{T u}\right)\right] \\
\times \exp \left[-\frac{i a u}{b}-\frac{T i u^{3}}{3 b s^{2}}\right] .
\end{gathered}
$$

The assignment of a small imaginary part to the coefficient $T$ appears arbitrary. It may be justified mathematically by an extension of Brownell's method, [15] which uses the complex Laplace transform with real coeffients and appropriate contours off the real axis in the complex $s$-plane. Here we employ the operationally simpler, and presumably equivalent, method of complex coefficients and a real Laplace transform variable.

From eqs (62), (64), and (67), and the convolution theorem for Fourier transforms, we obtain

$$
\begin{gathered}
j(x, s)=\frac{1}{2} a i(\pi i T)^{-1 / 2} \int_{0}^{\infty} d w w^{-1 / 2} \exp \left(-i a w-\frac{T i b^{2} w^{3}}{3 s^{2}}\right) \\
\times \int_{-\infty}^{\infty} d v y(v, 0) \exp (-i b v w)\left(1+\frac{b v}{a}\right) \\
\times \exp \left\{\frac{\mathrm{i}}{4}\left[\frac{T b^{2} w^{3}}{s^{2}}-2 b w(x-v)+\frac{s^{2}(x-v)^{2}}{T w}\right]\right\}
\end{gathered}
$$

where $w=b v$. At this point we may discard terms which are explicity $O\left(b^{z}\right)$ and rearrange, with the result that

$$
\begin{gathered}
j(x, s)=\frac{1}{2} a i(\pi i T)^{-1 / 2} \int_{-\infty}^{\infty} d v y(v, 0)\left(1+\frac{b v}{a}\right) \\
\times b_{b}^{\infty} d w w^{-1 / 2} \exp \left[-i a w-\frac{\mathrm{i}}{2} b u(x+v)+i s^{2}(x-v)^{2} / 4 T w\right] .
\end{gathered}
$$


To evaluate the $w$-integral, we make the substitution $w=z^{2}$. The relation [22]

$$
\int_{0}^{\infty} e^{-\alpha z^{2}-\beta / z^{2}} d z=\frac{1}{2}\left(\frac{\pi}{\alpha}\right)^{1 / 2} e^{-2(\alpha \beta)^{1 / 2}}
$$

holds for all $\alpha, \beta$ such that $R e(\alpha)>0$ and $R e(\beta)>0$. To apply this relation to eq (69), in addition to the small negative imaginary term added to $T$, a small positive imaginary term must be added to $a$. Then eq (69) reduces to

$$
\begin{aligned}
f(x, s) & =\frac{1}{2}(a / T)^{1 / 2} \int_{-\infty}^{\infty} d v y(v, 0)\left(1+\frac{b v}{a}\right)\left[1+\frac{b}{2 a}(x+v)\right]^{-1 / 2} \\
& \times \exp \left\{-s|x-v|\left[\left(a+\frac{1}{2} b[x+v]\right) / T\right]^{1 / 2}\right\} .
\end{aligned}
$$

At this point it is evident, by inspection, that the uniform limit $b \rightarrow 0+$ recovers the uniform solution, eq (33), where $c=(T / a)^{1 / 2}$.

To find the $P$-space solution, we use the relation

$$
\mathcal{L}^{-1}\left\{e^{-\alpha s}\right\}=\delta(t-\alpha)
$$

where $\mathcal{L}$ is the Laplace transform operator. This holds for real positive $\alpha$, and hence is applicable provided that

$$
(x+v)>-\frac{2 a}{b}
$$

Thus we see that the concerns raised in section 2.2 about the absence of explicit boundaries are built into the mathematics of the solution; an inverse Laplace transform cannot be performed for large negative $x$, where the differential equation becomes physical nonsense.

With this proviso, the $P$-space solution is

$$
\begin{aligned}
y(x, t)= & \frac{1}{2}(a / T)^{1 / 2} \int_{-\infty}^{\infty} d v y(v, 0)\left(1+\frac{b v}{a}\right)\left[1+\frac{b}{2 a}(x+v)\right]^{-1 / 2} \\
& \delta\left(t-|x-v|\left[a+\frac{1}{2} b(x+v)\right]^{1 / 2} / T\right)
\end{aligned}
$$

which, to leading order in nonuniformity, may be reduced to the more suggestive form,

$$
\begin{aligned}
y(x, t) & =\frac{1}{2}\left(1-\frac{b c t}{4 a}\right) y\left(x-c t\left[1-\frac{b}{4 a}(2 x-c t)\right], 0\right) \\
& +\frac{1}{2}\left(1+\frac{b c t}{4 a}\right) y\left(x+c t\left[1-\frac{b}{4 a}(2 x+c t)\right], 0\right)
\end{aligned}
$$

where $c=(T / a)^{1 / 2}$, i.e., two moving pulses. It is easy to show that, through terms linear in $b$, the pulses separately satisfy the original differential equation, eqs (58) and (59).

The $F$-space solution may be derived from eq $(75)$ and, to leading order in nonuniformity, can be represented in a similarly suggestive form,

$$
\begin{aligned}
\bar{y}(k, t)= & \frac{1}{2}\left(1-\frac{3 b c t}{4 a}\right) \bar{y}\left(k\left[1-\frac{b c t}{2 a}\right], 0\right) \exp \left[-i c\left(1-\frac{b c t}{4 a}\right) k t\right] \\
& +\frac{1}{2}\left(1+\frac{3 b c t}{4 a}\right) \bar{y}\left(k\left[1+\frac{b c t}{2 a}\right], 0\right) \exp \left[i c\left(1+\frac{b c t}{4 a}\right) k t\right],
\end{aligned}
$$

cf. eq (32).

Since eq (76) holds only for small times (cf. table 1), and there is no damping for the unperturbed solution, it is not meaningful to construct a dynamic structure factor for this case. 


\subsection{Interpretation}

It is at this point easily seen that the solution has the proper physical behavior expected on intuitive grounds. The first term of eq (75) is the rightward moving pulse. Since $b$ is positive, the wave speed decreases to the right, and, as it should, the pulse moves more slowly as time progresses. The factor $\left(1-\frac{b c t}{4 a}\right)$ indicates that the pulse size decreases with time, as it should since it moves into a region of greater inertia. In addition, the width of the pulse gets smaller in time by a factor $\left(1+\frac{b c t}{2 a}\right)^{-1}$, because the front end of the pulse moves more slowly than the back end. Conversely, the leftward moving pulse, or second term of eq (75), gets larger and wider and moves faster with time.

The $F$-space solution, eq (76), may be interpreted similarly. The rightward pulse, the first term, propagates with a decreasing time-dependent effective speed of $c\left(1-\frac{b c t}{4 a}\right)$. Due to Heisenberg's inequality, [21] the rightward pulse in $F$-space gets wider in time. To compensate, so that Parseval's theorem is satisfied, the factor in front must decrease more rapidly with time than the corresponding $P$-space factor. The converse observations apply to the leftward pulse.

We reiterate that it is apparently not possible to go directly from the $F L$-space solution, eq (62), to the leading-order $F$-space solution, eq (76), by means of an inverse Laplace transform. The concept of a nonuniformity expansion, as discussed in section $2 . \mathrm{I}$, appears to be meaningful only in $P$-space and $L$-space, not in $F$-space or $F L$-space. Hence we must follow the circuitous route shown in figure 1.

\section{Nonuniform diffusion equation}

\subsection{The differential equation}

In the study of the nonuniform diffusion equation, we must at the outset distinguish material diffusion from diffusion of a thermal pulse. The equation for material diffusion in one dimension is [23]

$$
\frac{\partial}{\partial t} y(x, t)-\frac{\partial}{\partial x}\left[D(x) \frac{\partial}{\partial x} y(x, t)\right]=0
$$

and the case of interest to us is

$$
D(x)=D_{a}+D_{1} x
$$

where $D_{1}$ is small.

Diffusion of a thermal pulse in one dimension, [24] which corresponds more closely to the RayleighBrillouin problem, is characterized by a thermal conductivity $\lambda(x)$ and a heat capacity per unit length $\sigma(x)$.
The differential equation is [24]

$$
\frac{\partial}{\partial t} y(x, t)-\frac{1}{\sigma(x)} \frac{\partial}{\partial x}\left[\lambda(x) \frac{\partial}{\partial x} y(x, t)\right]=0
$$

and the cases of interest are

$$
\begin{aligned}
& \lambda=\lambda_{0}+\lambda_{1} x \\
& \sigma=\sigma_{a}+\sigma_{1} x
\end{aligned}
$$

where $\lambda_{1}$ and $\sigma_{1}$ are small.

To linear order in nonuniformity, we may represent both equations by the convenient form

$$
(a-b x) \frac{\partial y}{\partial t}-c \frac{\partial^{2} y}{\partial x^{2}}+\gamma b \frac{\partial y}{\partial x}=0
$$


where, for material diffusion,

$$
\begin{aligned}
& \frac{c}{a}=D_{o} \\
& \frac{b}{a}=D_{1} / D_{o} \\
& \gamma=-D_{o}
\end{aligned}
$$

and for diffusion of a thermal pulse,

$$
\begin{aligned}
& a=\sigma_{o} \lambda_{o} \\
& b=\sigma_{o} \lambda_{1}-\lambda_{o} \sigma_{1} \\
& c=\lambda_{o}^{2} \\
& \gamma=\lambda_{o} \lambda_{1} / b .
\end{aligned}
$$

The equation thus is characterized by two nonuniformity parameters $b$ and $\gamma$, and a single small parameter $b$.

\subsection{Properties of the solution}

Before embarking on a derivation of the solution to eq (81), it is of interest to examine certain properties of the solution which follow directly from the differential equation itself. We multiply both sides of eq (81) by $(1+b x / a)$, discard terms quadratic in $b$, and integrate over $x$ from $-\infty$ to $\infty$. The result is

$$
\frac{\partial}{\partial t} \int_{-\infty}^{\infty} y(x, t) d x=0
$$

so that the norm $\|y\|$ considered previously, eq (24), is a constant of the motion. For material diffusion, eq (77), the norm is constant for any possible $D(x)$, a consequence of the conservation of diffusing material. For thermal diffusion, eq (79), the above norm is not constant in general, but is so to first order in nonuniformity.

If we consider pulses with unit norm, the central position is given by

$$
\langle x\rangle=\int_{-\infty}^{\infty} x y(x, t) d x .
$$

Multiplying the differential equation by $x$ and integrating yields

$$
\frac{\partial}{\partial t}\langle x\rangle=\frac{2 c b}{a^{2}}+\frac{\gamma b}{a} \text {. }
$$

Physically, for positive $\lambda_{1}$ and negative $\sigma_{1}$ the diffusion coefficient effectively increases with $x$. Here $b$ and $\gamma$ are positive, so the average position of the pulse drifts to the right. In the converse situation, the pulse drifts to the left.

A similar calculation yields the time derivative of the mean-square displacement,

$$
\frac{\partial}{\partial t}\left\langle x^{2}\right\rangle=2 \frac{c}{a}+\left(\frac{2 \gamma b}{a}+\frac{6 b c}{a^{2}}\right)<x>
$$

and the mean-square width,

$$
\frac{\partial}{\partial t}\left\langle\Delta x^{2}\right\rangle=\frac{\partial}{\partial t}\left[\left\langle x^{2}\right\rangle-\langle x\rangle^{2}\right]=\frac{2 c}{a}+\frac{2 c b}{a^{2}}\langle x\rangle
$$

so that the increase in width with time is independent of $\gamma$. 


\subsection{Solution}

The solution of the nonuniform diffusion equation closely parallels that of the nonuniform wave equation. The differential equation in $F L$-space is

$$
\begin{gathered}
\frac{\partial}{\partial k} \tilde{y}(k, s)+\left[\frac{a i}{b s}+\frac{c k^{2} i}{b s}-\frac{\gamma k}{s}\right] \tilde{y}(k, s)= \\
\frac{1}{s} \frac{\partial}{\partial k} \bar{y}(k, 0)+\frac{a i}{b s} \tilde{y}(k, 0) .
\end{gathered}
$$

Again, this linear differential equation in $k$ is solved by the integrating factor technique, with the boundary condition $k_{1}=\infty$. The result is

$$
\begin{aligned}
\tilde{y}(k, s) & =-\int_{o}^{\infty} d u\left[\frac{a i}{b s} \bar{y}(u+k, 0)+\frac{1}{s} \frac{\partial}{\partial k} \bar{y}(u+k, 0)\right] \\
& \times \exp \left[\frac{a i u}{b}+\frac{c i}{3 b s} u\left(u^{2}+3 u k+3 k^{2}\right)-\frac{\gamma}{2 s} u(u+2 k)\right] .
\end{aligned}
$$

Without loss of generality we consider only positive $b$. The convolution theorem is used to transform the solution to $\mathrm{L}$-space. We are required to add a small positive imaginary part to $c$. The result is

$$
\begin{aligned}
\hat{y}(x, s) & =\frac{1}{2} a(b s i c \pi)^{-1 / 2} \int_{0}^{\infty} u^{-1 / 2} d u \exp \left[\frac{a i u}{b}+\frac{c i u^{3}}{3 b s}-\frac{\gamma u^{2}}{2 s}\right] \\
& \times \int_{-\infty}^{\infty} d v y(v, 0)\left[1-\frac{b v}{a}\right] \exp [-i u v] \\
& \times \exp \left[-\frac{c u^{3} i}{4 b s}-\frac{i b s}{4 c u}(x-v)^{2}-\frac{i u}{2}(x-v)\right. \\
& \left.+\frac{b \gamma^{2} u i}{4 c s^{2}}+\frac{\gamma b}{2 c}(x-v)+\frac{\gamma u^{2}}{2 s}\right] .
\end{aligned}
$$

Again we make the substitution $u=b w$ and then discard all $o\left(b^{2}\right)$ terms. We next explicitly perform the w-integral by means of the substitution $w=z^{2}$ and eq (70). It is necessary to assign a small positive imaginary element to a. The result of these operations is

$$
\begin{aligned}
\hat{y}(x, s) & =\frac{1}{2}(a / c s)^{1 / 2} \int_{-\infty}^{\infty} d v y(v, 0)\left[1-\frac{b v}{a}\right]\left[1-\frac{b}{2 a}(x+v)\right]^{-1 / 2} \\
& \times \exp \left[\frac{\gamma b}{2 c}(x-v)\right] \exp -\left\{(s a / c)^{1 / 2}|x-v|\left[1-\frac{b}{2 a}(x+v)\right]^{1 / 2}\right\} .
\end{aligned}
$$

At this point it is easily seen that the uniform limit, $b-0+$, recovers the uniform solution, eq (4l) with $D$ $=c / a$.

To determine the $P$-space solution, we use the relation

$$
\mathcal{L}^{-1}\left\{s^{-1 / 2} e^{-\alpha s^{1 / 2}}\right\}=(\pi t)^{-1 / 2} e^{-\alpha^{2 / 4 t}}
$$

This equation is applicable for real positive $\alpha$, which requires that

$$
x+v<\frac{2 a}{b} .
$$

Once again, it is built into the mathematics that a solution does not exist for large displacements where the original differential equation becomes unphysical. 
The $P$-space solution initially is most conveniently represented in kernel form,

$$
y(x, t)=\int_{-\infty}^{\infty} y(v, 0) K(x, v ; t) d v
$$

where, to linear order in $b$,

$$
\begin{aligned}
K(x, v ; t) & =\frac{1}{2}\left(\pi D_{o} t\right)^{-1 / 2}\left[1+\frac{b}{4 a}(x-3 v)+\frac{\gamma b}{2 c}(x-v)\right] \\
& \times \exp \left\{-(x-v)^{2}\left[1-\frac{b}{2 a}(x+v)\right] / 4 D_{o} t\right\}
\end{aligned}
$$

and $D_{o}=c / a$ is the coefficient of diffusion in the uniform limit.

However, there is a severe mathematical difficulty with eqs (95) and (96) in their present form; namely, the integral of eq (95) does not exist as written. There is an essential difference between the solutions of the undamped wave equation and the diffusion equation. For the undamped wave equation, if the initial pulse is strictly localized around the origin, i.e., $y(x, 0)=0$ identically for $|x|>w_{0} / 2$, then, for any large distance $L, y(L, t)$ remains strictly zero until some finite time of the order $L / c$. However, as governed by the diffusion equation, a similar localized pulse causes a finite displacement at $L$ after any time interval, however small, as an infinite tail is instantaneously created of the form $\exp \left(-x^{2}\right)$ at large $x$. Then, since the kernel of eq (96) goes as $\exp \left(+v^{3}\right)$ for large $v$, the integral of eq (95) is formally infinite after any two successive finite time steps.

Physically, however, it is not possible for a pulse to diffuse over large distances instantaneously; this phenomenon should be interpreted as a mathematical artifact. We believe the proper way to interpret eqs (95) and (96) physically is to remove this artifact in the following manner. The kernel of the integrand is a distorted Gaussian function integrated over an undistorted measure. A transformation is made so that the kernel becomes an undistorted Gaussian integrated over a distorted measure. Specifically, we define the new measure

$$
u^{2}=(x-v)^{2}\left[1-\frac{b}{2 a}(x+v)\right]
$$

and

$$
u=(v-x)\left[1-\frac{b}{4 a}(x+v)\right]+O\left(b^{2}\right)
$$

Then, if terms higher than linear order in $b$ are discarded, eq (95) becomes

$$
\begin{gathered}
y(x, t)=\frac{1}{2}\left(\pi D_{o} t\right)^{-1 / 2} \int_{-\infty}^{\infty} d u y\left(x+u\left[1+\frac{b}{4 a}(2 x+u)\right], 0\right) \\
{\left[1-\frac{b u}{4 a}-\frac{\gamma b u}{2 c}\right] e^{-u^{2} / 4 D_{o} t} .}
\end{gathered}
$$

The major formal change between eq (95) and eq (99) is the upper limit of infinity on the $u$ integration, whereas, from eq (97), as $v \rightarrow+\infty, u$ becomes, formally, infinite and imaginary. The objective of the transformation is to suppress the unphysical behavior for $x>a / b$ where the diffusion is mathematically negative. Equation (99) is a well-behaved solution to linear order in nonuniformity which connects smoothly to the uniform case.

To check the solution, we first verify that $K(x, v ; t)$ satisfies the boundary condition

which is equivalent to

$$
K(x, v ; 0)=\delta(x-v)
$$

$$
K(x, v ; 0)=0 \quad[x \neq v]
$$

and

$$
\int_{-\infty}^{\infty} K(x, v ; 0) d v=1 .
$$


Equation (101) is verified by inspection, and eq (102) is seen to be valid in the sense of the transformation from eq (96) to eq (99). Finally, it is straightforward to show that the kernel should and does satisfy the differential equation

$$
c \frac{\partial^{2}}{\partial x^{2}} K(x, v ; t)-(a-b x) \frac{\partial}{\partial t} K(x, v ; t)-\gamma b \frac{\partial}{\partial x} K(x, v ; t)=0
$$

through linear order in $b$.

The $F$-space solution may be derived by expanding y in the integrand of eq (99) through linear order in $b$, rearranging the integrand into sums of terms of the form $f(u) g(x-u)$, and applying the convolution theorem. The result is

$$
\begin{gathered}
\bar{y}(k, t)=\bar{y}(k, 0) e^{-D_{0} k^{2} t}\left[1-i\left(\frac{2 b}{a}+\frac{\gamma b}{c}\right) D_{o} k t+i \frac{b}{a} D_{a}^{2} k^{3} t^{2}\right] \\
-\left[\frac{\partial}{\partial k} \bar{y}(k, 0)\right] e^{-D_{o} k_{t}} i \frac{b}{a} D_{o} k^{2} t
\end{gathered}
$$

This solution satisfies the initial condition by inspection. It is also straightforward to check that it satifies the differential equation, eq $(81)$ transformed to $F$-space, through linear order in $b$.

We proceed to calculate the dynamic structure factor, the time Fourier transform of the intermediate scattering function $F(k, t)$ (cf. eqs (2-3)). The solution of the initial value problem in principle yields $F(k, t)$ only for positive times. We employ the usual convention for a time correlation function that for negative times [25] the function is defined to be

$$
F(\mathrm{k}, \mathrm{t})=F^{*}(k,-t)
$$

where the asterisk denotes a complex conjugate, in which case

$$
S(k, \omega)=2 \operatorname{Re} \int_{0}^{\infty} e^{-i \omega t} F(k, t) d t
$$

Applying eq (106) to our solution yields the result

$$
\begin{aligned}
& S(k, \omega)=\left\langle\bar{y}(-k, 0) \bar{y}(k, 0)>\left\{\frac{2 D_{o} k^{2}}{\omega^{2}+\left(D_{o} k^{2}\right)^{2}}-\left(\frac{2 b}{a}+\frac{\gamma b}{c}\right) \frac{4 D_{o}^{2} \omega k^{3}}{\left[\omega^{2}+\left(D_{o} k^{2}\right)^{2}\right]^{2}}\right.\right. \\
& \left.+2 \frac{b}{a} D_{0}^{2} k^{3} \frac{\omega^{3}-3 \omega\left(D_{o} k^{2}\right)^{2}}{\left[\omega^{2}+\left(D_{o} k^{2}\right)^{2}\right]^{3}}\right\}-<y(-k, 0) \frac{d}{d k} \bar{y}(k, 0)>\frac{4 b}{a} \frac{D_{o}^{2} \omega k^{4}}{\left[\omega^{2}+\left(D_{o} k^{2}\right)^{2}\right]^{2}} .
\end{aligned}
$$

\subsection{Interpretation}

The most important physical features of the solution to the nonuniform diffusion equation may be given a simple interpretation as a "Doppler shift" similar to that of the uniform damped wave equation. In particular, eq (86) shows that the diffusion pulse, on the average, drifts in the direction of increasing diffusion with a velocity $c_{D}$ given by

$$
c_{D}=\frac{2 c b}{a^{2}}+\frac{\gamma b}{a}
$$

The uniform damped wave pulse undergoes a similar motion with the wave speed $\mathrm{c}$ and an "effective diffusion rate" of $\Gamma$. By a naive analogy we would expect that the F-space nonuniform solution is

$$
\bar{y}(k, t)=\bar{y}(k, 0) e^{-D_{0} k^{2} t} e^{-i c_{D} k t}
$$


which, through first order in $c_{D}$, yields the first two terms of eq (104). By similar reasoning we would obtain the dynamic structure factor

$$
S(k, \omega)=<-\bar{y}(-k, 0) \bar{y}(k, 0)>\frac{2 D_{o} k^{2}}{\left(\omega+c_{D} k\right)^{2}+\left(D_{o} k^{2}\right)^{2}}
$$

which similarly accounts for the first two terms of eq $(107)$ through $0\left(c_{D}\right)$.

The third term of eq (107) gives a rather complicated change of shape of the "Rayleigh line" not predicted by the above heuristic arguments. The term oscillates several times and is qualitatively akin to the third frequency derivative of the primary Lorentzian function, so it alters, in leading order, neither the mean frequency nor the width.

The ensemble average of the fourth term in eq (107) vanishes for a uniform system because of invariance under a parity transformation. This term is somewhat difficult to interpret, but, when averaged over an appropriate ensemble, is presumably no more than first order in nonuniformity. Hence the entire term presumably yields a contribution second order in nonuniformity and may be neglected.

\section{Nonuniform damped wave equation}

\subsection{The differential equation}

We first seek the appropriate one-dimensional nonuniform analog to the equation, first derived by Stokes, for the propagation of a plane sound wave in an isotropic viscous medium, [26]

$$
e \frac{\partial^{2} \zeta}{\partial t^{2}}-x \frac{\partial^{2} \zeta}{\partial x^{2}}-\frac{4}{3} \mu \frac{\partial^{2}}{\partial x^{2}} \frac{\partial \zeta}{\partial t}=0
$$

cf. eq (46), where $\varrho$ is the density, $\zeta$ the displacement, $x$ the effective bulk modulus, and $\mu$ the viscosity. This corresponds to the neglect of coupling between density and temperature fluctuations in the RayleighBrillouin scattering problem. [11]

The following model, though somewhat contrived, appears to be the one-dimensional analog to the above with the appropriate properties. The viscous term corresponds in three dimensions to an off-diagonal term in the pressure tensor, which, crudely speaking, represents a torque per unit volume. In one dimension, this would be a torque per unit length.

We consider a stretched string with position-dependent mass per unit length $\mathrm{\varrho}(x)$. We imagine that, as the string vibrates transversely, it traverses a viscous medium which exerts a torque $\Delta \mathrm{L}$ on a length element $\Delta x$. On each element $\Delta x$ there is a force $F(x)$, perpendicular to the string, acting at one end and an equal and opposite force acting on the other end. Thus

$$
\Delta L=[F(x)+F(x+\Delta x)] \Delta x .
$$

We further assume that the force is proportional to the spatial derivative of the velocity of the segment, the analog of the three-dimensional velocity gradient

$$
F(x)=2 \mu(x) \frac{\partial}{\partial x} \frac{\partial y}{\partial t}
$$

where $\mu(x)$ is analogous to viscosity and may be position-dependent.

Next we generalize the standard derivation [27] of the wave equation for a stretched string under tension $T$ by the inclusion of this new force. As usual it is assumed that the angle between a vibrating segment of the string and the equilibrium direction is sufficiently small that its sine and tangent are equivalent. Applying Newton's second law to the segment of the string from $x_{1}$ to $x_{2}$ yields 


$$
\begin{aligned}
\int_{x_{1}}^{x_{2}} \varrho(x) \frac{\partial^{2} y}{\partial t^{2}} d x & =\int_{x_{1}}^{x_{2}} \frac{\partial}{\partial x}\left[T \frac{\partial y}{\partial x}\right] d x \\
& +2 \int_{x_{1}}^{x_{2}} \frac{\partial}{\partial x}\left[\mu(x) \frac{\partial}{\partial x} \frac{\partial y}{\partial t}\right]
\end{aligned}
$$

or, since $x_{1}$ and $x_{2}$ are arbitrary,

$$
\varrho(x) \frac{\partial^{2} y}{\partial t^{2}}-T \frac{\partial^{2} y}{\partial x^{2}}-2-\frac{\partial}{\partial x} \mu(x) \frac{\partial}{\partial x} \frac{\partial y}{\partial t}=0 .
$$

This is the particular nonuniform damped wave equation to be analyzed here. We are interested in the special case

$$
\begin{aligned}
& \varrho(x)=a+b x \\
& \mu(x)=\mu+\beta x
\end{aligned}
$$

where $b$ and $\beta$ are small.

\subsection{Solution}

The solution of the nonuniform damped wave equation closely parallels those of the undamped wave and diffusion equations. The equation in FL-space is

$$
\begin{aligned}
a s^{2} \tilde{y} & +i b s^{2} \frac{\partial \tilde{y}}{\partial k}+T k^{2} \tilde{y}+2 \mu s k^{2} \tilde{y}+2 \beta i k s \tilde{y}+2 \beta i k^{2} s \frac{\partial \tilde{y}}{\partial k}=a s \bar{y}(k, 0) \\
& +i b s \frac{\partial}{\partial k} \bar{y}(k, 0)+2 \mu k^{2} \bar{y}(k, 0)+2 \beta i k \bar{y}(k, 0)+2 \beta i k^{2} \frac{\partial}{\partial k} \bar{y}(k, 0) .
\end{aligned}
$$

As before, this linear differential equation may be solved by the integrating factor technique, [20] However, at this point we anticipate a mathematical difficulty. In previous examples, where the linear differential equation had the form

$$
\frac{\partial \tilde{y}}{\partial k}+f(k) \tilde{y}=g(k)
$$

the variable $f(k)$ had the form of a quadratic polynomial in $k$. This property made possible the subsequent inverse Fourier transformation (from $F L$-space to $L$-space) by means of eq (65). Upon examination of eq (118), we note that $f(k)$ is quadratic if and only if $\beta=0$. We shall therefore set $\beta=0$ in the remainder of this section. This corresponds to uniform "viscosity" with nonuniform density, or a particular correlated case of nonuniform wave speed and wave damping.

Again we use the boundary condition $k_{1}=\infty$, and make the substitution $k^{\prime}=u+k$ [cf. eqs (61)-(62)]. The $F L$-space solution is

$$
\begin{aligned}
\tilde{y}(k, s) & =\int_{0}^{\infty} d u\left[\frac{a i}{b s} \bar{y}(u+k, 0)+\frac{2 i \mu(u+k)^{2}}{b s^{2}} \bar{y}(u+k, 0)\right. \\
& \left.-\frac{1}{s} \frac{\partial}{\partial k} \bar{y}(u+k, 0)\right] \exp \left[-\frac{i a u}{b}-\frac{(T+2 \mu s) i}{3 b s^{2}} u\left(u^{2}+3 k u+3 k^{2}\right)\right]
\end{aligned}
$$


Without loss of generality, we consider only positive $b$. The convolution theorem is then used to derive the $\mathrm{L}$-space solution. Once again, it is necessary to assign a small negative imaginary component to $\mathrm{T}$. The result is

$$
\begin{aligned}
\hat{y}(x, s) & =\frac{a i}{2}[\pi b i(T+2 \mu s)]^{-1 / 2} \int_{o}^{\infty} d u u^{-1 / 2} \exp \left[-\frac{i a u}{b}\right. \\
& \left.-\frac{(T+2 \mu s) i u^{3}}{3 b s^{2}}\right] \int_{-\infty}^{\infty} d v\left[y(v, 0)\left(1+\frac{b v}{a}\right)-\frac{2 \mu}{a s} \frac{\partial^{2}}{\partial v^{2}} y(v, 0)\right] \\
& \times \exp (-i u v) \exp \left\{\frac{\mathrm{i}}{4}\left[\frac{(T+2 \mu s) u^{3}}{b s^{2}}-2 u(x-v)+\frac{b s^{2}(x-v)^{2}}{(T+2 \mu s) u}\right]\right\} .
\end{aligned}
$$

As in the undamped wave solution, we make the substition $u=b w$ and discard terms explicitly $O\left(b^{2}\right)$. We then let $w=z^{2}$ and apply eq (70), after assigning a small positive imaginary part to a. The result is

$$
\begin{aligned}
\hat{y}(x, s)=1 / 2 a^{1 / 2}(T+2 \mu s)^{-1 / 2} \int_{-\infty}^{\infty} d v\left[y(v, 0)\left[1+\frac{b v}{a}\right]-\frac{? \mu}{a s} \frac{\partial^{2}}{\partial v^{2}} y(v, 0)\right] \\
{\left[1+\frac{b}{2 a}(x+v)\right]^{-1 / 2} \exp \left\{-s|x-v|\left[1+\frac{b}{2 a}(x+v)\right]^{1 / 2}[a /(T+2 \mu s)]^{1 / 2}\right\} . }
\end{aligned}
$$

In the uniform limit, $b \rightarrow 0+$, we recover eq (52), with $\Gamma=\mu / a$ and $c=(T / a)^{1 / 2}$.

At this point we encounter the problem of dispersion discussed in section 2.3. Since there is no general $P$-space solution to the uniform damped wave equation in terms of elementary functions, we cannot expect to find an explicit $P$-space solution to the nonuniform equation either (to all orders in dispersion). A formal expression may be written,

$$
\begin{aligned}
& y(x, t)=1 / 2 \int_{-\infty}^{\infty} d v\left[1+\frac{b}{2 a}(x+v)\right]^{-1 / 2}\left[y ( v , 0 ) ( 1 + \frac { b v } { a } ) \mathcal { L } ^ { - 1 } \left\{\left(c^{2}+2 \Gamma s\right)^{-1 / 2}\right.\right. \\
& \left.\exp \left[-s|x-v|\left[1+\frac{b}{2 a}(x+v)\right]^{1 / 2}\left(c^{2}+2 \Gamma s\right)^{-1 / 2}\right]\right\}-2 \Gamma \frac{\partial^{2}}{\partial v^{2}} y(v, 0) \mathcal{L}^{-1}\left\{s^{-1}\right. \\
& \left.\left.\left(c^{2}+2 \Gamma s\right)^{-1 / 2} \exp \left[-s|x-v|\left[1+\frac{b}{2 a}(x+v)\right]^{1 / 2}\left(c^{2}+2 \Gamma s\right)^{-1 / 2}\right]\right\}\right]
\end{aligned}
$$

where $\mathcal{L}^{-1}$ is the inverse Laplace transform operator. However, this form is not particularly illuminating physically.

To display a more transparent solution, we assume that there exists an operator $\tilde{\mathcal{L}}^{-1}$ which simultaneously performs an inverse Laplace transformation and projects out the leading term in a dispersion expansion. For example, on comparing eqs (52) and (55), we see that such an operator should effect the transformation

$$
\begin{gathered}
\tilde{\mathcal{L}}^{-1}\left\{\left(c^{2}+2 \Gamma s\right)^{-1 / 2} \exp \left[-s|x-v|\left(c^{2}+2 \Gamma s\right)^{-1 / 2}\right]\right\} \\
=1 / 2(\pi \Gamma t)^{-1 / 2}\left[e^{-(x-v+c t)^{2} / 4 \Gamma t}+e^{-(x-v-c t)^{2} / 4 \Gamma t}\right] .
\end{gathered}
$$

We further assume that $\widetilde{\mathcal{L}}^{-1}$ obeys the change of scale property of Laplace transforms, namely, if

$$
\mathcal{L}^{1}\{f(s)\}=F(t)
$$

then

$$
\tilde{L}^{-1}\{f(\alpha s)\}=\frac{1}{\alpha} F\left(\frac{t}{\alpha}\right)
$$

at least providing $\alpha$ depends only on position and nonuniformity parameters. The above is, in essence, a statement of the independence of nonuniformity and dispersion expansions. 
The second term of eq (116) is of higher order in dispersion than the first term and may be neglected. On replacing $\mathcal{L}^{-1}$ by $\mathcal{L}^{-1}$ in eq (123) and using eqs (124)-(126), we obtain the solution

$$
\begin{gathered}
y(x, t)=1 / 4(\pi \Gamma t)^{-1 / 2} \int_{-\infty}^{\infty} d v y(v, 0)\left[1+\frac{b v}{a}\right]\left[1+\frac{b}{2 a}(x+v)\right]^{-1 / 2} \\
{\left[\operatorname { e x p } \left\{-\left[c t-\left(1+\frac{b}{2 a}[x+v]^{1 / 2}(x-v)\right]^{2} / 4 \Gamma t\right\}\right.\right.} \\
+\exp \left\{-\left[c t+\left(1+\frac{b}{2 a}[x+v)^{1 / 2}(x-v)\right]^{2} / 4 \Gamma t\right\}\right]
\end{gathered}
$$

which bears a strong similarity to the nonuniform diffusion solution, eqs (95)-(96), for $\gamma=0$.

As in the case of diffusion, we encounter problems with divergences in the integrals due to mathematical. ly instantaneous propagation of the pulse to points at large distances from the origin, where the original differential equation makes no physical sense. We again surmount the difficulty by transforming to a distorted measure in which the Gaussians of the kernel are undistorted. The new measure is

$$
u=(x-v)\left[1+\frac{b}{2 a}(x+v)\right]^{1 / 2} .
$$

Then a calculation, discarding terms higher than linear order in $b$, analogous to that of diffusion yields

$$
\begin{gathered}
y(x, t)=1 / 4(\pi \Gamma t)^{-1 / 2} \int_{-\infty}^{\infty} d u y\left[x-u\left(1-\frac{b}{4 a}[2 x-u], 0\right]\right. \\
\left(1-\frac{b u}{4 a}\right)\left[e^{-(u-c t)^{2} / 4 \Gamma t}+e^{-(u+c t)^{2} / 4 \Gamma t}\right] .
\end{gathered}
$$

Since

$$
\lim _{\Gamma \rightarrow 0} 1 / 2(\pi \Gamma t)^{-1 / 2} e^{-(u \pm c t)^{2} / 4 \Gamma t}=\delta(u \pm c t)
$$

it is easily checked that, in the limit of zero damping, the nonuniform undamped wave equation solution, eq (75), is recovered.

We recall that in the present problem there are two small expansion parameters, namely, nonuniformity and dispersion. Since the latter is defined in terms of $F$-space variables, there is no natural means of checking that the $P$-space solution satisfies the differential equation.

The $F$-space solution may be derived by expanding y in the integrand of eq (129) through linear order in $\mathrm{b}$, rearranging the integrand into sums of terms of the form $f(u+c t) g(x-u)$, and repeatedly applying the convolution theorem. The end result is rather lengthy and is best expressed as the sum of a rightward and a leftward pulse.

$$
\bar{y}(k, t)=\bar{y}_{r}(k, t)+\bar{y}_{t}(k, t)
$$

where

$$
\begin{gathered}
\bar{y}_{r}(k, t)=\bar{y}(k, 0) e^{-\Gamma k^{2} t} e^{-i c k t} \\
{\left[\frac{1}{2}+\frac{b}{a} i \Gamma k t-\frac{3 b}{8 a} c t-\frac{b}{2 a} i \Gamma^{2} k^{3} t^{2}+\frac{b}{2 a} \Gamma c k^{2} t^{2}+\frac{b}{8 a} i c^{2} k t^{2}\right]} \\
+\left[\frac{\partial}{\partial k} \bar{y}(k, 0)\right] e^{-\Gamma k^{2} t} e^{-i c k t}\left[\frac{b}{2 a} i \Gamma k^{2} t-\frac{b}{4 a} c k t\right]
\end{gathered}
$$


linear order in dispersion, the term above containing $\Gamma^{2}$ may be discarded. 
The F-space transformation of the original differential equation, eqs (115-117) with $\beta=0$, is

$$
\frac{\partial^{2} \bar{y}}{\partial t^{2}}+\frac{b}{a} \mathrm{i} \frac{\partial}{\partial k} \frac{\partial^{2} \bar{y}}{\partial t^{2}}+k^{2} c^{2} \bar{y}+2 \Gamma k^{2} \frac{\partial \bar{y}}{\partial t}=0
$$

At this point it can be checked that the solution, eq (132), satisfies the differential equation, eq (133), if terms $O\left(b^{2}\right)$, second order in nonuniformity, and $O\left(\Gamma^{2}\right)$, second order in dispersion, are neglected.

The solution satisfies the initial value condition by inspection, but does not satisfy the condition on the initial time derivative. The latter, however, is not a failing of the nonuniformity analysis but, rather, a feature of the truncation of the dispersion expansion of the uniform damped wave solution. In particular, eq (51) is the correct solution to all orders in dispersion, whereas eq (54) satisfies the differential equation and initial value condition through first order in dispersion, but not the initial time derivative condition. A fully consistent first-order truncation of the dispersion expansion which includes the time derivative condition would retain the second term of eq (51) and replace $\left(c^{2}-\Gamma^{2} k^{2}\right)^{1 / 2}$ by $c$ throughout.

A similar difficulty appears in the standard treatment of Rayleigh-Brillouin scattering; compare, for example, eq (27) of ref. [11] with eq (97) of ref. [28]. Although the extra term required to make the initial time derivative vanish is in practice often neglected, for the sake of a fully consistent solution through first order in both dispersion and nonuniformity we shall find and include such terms here.

We note that eq (131) remains a solution through the required order if terms are added which are proportional to either $\Gamma$ times the solution with $\Gamma \rightarrow 0$ in the brackets of eq (132), or $b$ times the solution with $b \rightarrow$ 0 in the same brackets. It is straightforward to set up a trial solution with variable coefficients for the required additional terms, and solve for the coefficients subject to the constraints that the initial value vanish and the initial time derivative be equal and opposite to that of eqs (131)-(132). To obtain the desired result, a term

$\Delta \bar{y}_{n}(k, t)=\bar{y}(k, 0) e^{-\Gamma k^{2} t} e^{-i c k s}$

$$
\begin{gathered}
{\left[\frac{i \Gamma k}{c}\left(\frac{1}{2}-\frac{3 b}{8 a} c t+\frac{b}{8 a} i c^{2} k t^{2}\right)+\frac{5 \Gamma b}{8 c a}\right]} \\
+\left[\frac{\partial}{\partial k} \bar{y}(k, 0)\right] e^{-\Gamma k^{2} t} e^{-i c k t}\left[\frac{\Gamma k b}{4 c a}-\frac{i \Gamma b k^{2} t}{4 a}\right]
\end{gathered}
$$

must be added to $\bar{y}_{r}(k, t)$, and a term $\Delta \bar{y}_{\ell}(k, t)$, eq (134) with $c$ replaced by $-c$ throughout, must be added to $\bar{y}_{\ell}(k, t)$. We could, in a similar manner, impose a nonzero initial time derivative proportional to $\mathrm{b}$ and determined from external considerations, but will not do so here.

In the construction of the dynamic structure factor we will, for reasons decribed in section 5.4, neglect terms involving $\left(\frac{d}{d k} \bar{y}(k, 0)\right.$.) The resulting dynamic structure factor may be written as

$$
S(k, \omega)=S_{r}(k, \omega)+S_{f}(k, \omega)
$$

where

$$
\begin{aligned}
S_{r}(k, \omega) & =\left\langle\bar{y}(-k, 0) \bar{y}(k, 0)>\times\left[\left(1+\frac{5 \Gamma b}{8 c a}\right) \frac{\Gamma k^{2}}{(\omega+c k)^{2}+\left(\Gamma k^{2}\right)^{2}}\right.\right. \\
& +\frac{\Gamma k}{c} \frac{\omega+c k}{(\omega+c k)^{2}+\left(\Gamma k^{2}\right)^{2}}+\frac{5 b}{2 a} \Gamma^{2} k^{3} \frac{\omega+c k}{\left[(\omega+c k)^{2}+\left(\Gamma k^{2}\right)^{2}\right]^{2}} \\
& -\frac{3 b c}{4 a} \frac{\left(\Gamma k^{2}\right)^{2}-(\omega+c k)^{2}}{\left[(\omega+c k)^{2}+\left(\Gamma k^{2}\right)^{2}\right]^{2}}+\frac{3 b c}{2 a}\left(\Gamma k^{2}\right)^{2} \frac{\left(\Gamma k^{2}\right)^{2}-3(\omega+c k)^{2}}{\left[(\omega+c k)^{2}+\left(\Gamma k^{2}\right)^{2}\right]^{3}} \\
& \left.+\frac{b c^{2} k}{2 a} \frac{3(\omega+c k)\left(\Gamma k^{2}\right)^{2}-(\omega+c k)^{3}}{\left[(\omega+c k)^{2}+\left(\Gamma k^{2}\right)^{2}\right]^{3}}\right]
\end{aligned}
$$

and $S(k, \omega)$ is given by eq (136) with $c$ replaced by $-c$ throughout. 


\subsection{Interpretation}

In order to develop a physical understanding of the nonuniform damped wave equation solution, we first note that the effects of nonuniform speed and nonuniform damping are not independent and, in the special case considered here, tend to cancel each other. According to eqs. (115-117) with $b>0$ and $\beta=0$, both the wave speed and attenuation decrease to the right and increase to the left. Hence we expect the rightward pulse to undergo less damping, effectively, than in the uniform case. However, as has been seen explicitly with the nonuniform undamped wave solution, the decreasing wave speed attenuates the rightward pulse in a different sense by lowering its time-dependent integrated norm [eq (24)].

We have been careful to retain all terms linear in the nonuniformity and/or dispersion parameters. However, some terms in the end give much smaller contributions to $S(k, \omega)$ than others. For example, the conditions from table I on the validity of $S(k, \omega)$ together with the smallness of the dispersion parameter imply the inequalities

$$
\frac{b}{a k}<<\frac{\Gamma k}{c}<<1 \text {. }
$$

The rightward pulse (with our sign conventions) corresponds to the Brillouin line (Stokes line) centered at $\omega=-c k$. The additional factor $5 \Gamma b / 8 c a$ multiplying the primary Brillouin line [first term in eq (136)] is by (137) at least three orders of magnitude less than unity, and thus utterly negligible.

The remaining terms in eq (136) are conveniently classified quantitatively, to leading order, in terms of the shift in mean frequency or change in width they cause in the Brillouin line. Since the more commonly used definitions of mean position or width of a peak involve integrals which diverge for the Lorentzian function, we employ the following alternate definitions, for mean frequency,

$$
\bar{\omega}=\int_{-\infty}^{\infty} \omega[f(\omega)]^{2} d \omega / \int_{-\infty}^{\infty}[f(\omega)]^{2} d \omega
$$

and for width of the line,

$$
(\Delta \omega)^{2}=\int_{-\infty}^{\infty}(\omega-\bar{\omega})^{2}[f(\omega)]^{2} d \omega / \int_{-\infty}^{\infty}[f(\omega)]^{2} d \omega
$$

For the primary Brillouin line eq (139) gives, as usually defined, $\Delta \omega=\Gamma k^{2}$. The precise definitions are not important but the above ones serve as convenient measures of changes in the spectrum for purposes of discussion.

The second term of eq (136) is antisymmetric about $\omega=-c k$ and corresponds to the well-known firstorder dispersion term in the Rayleigh-Brillouin spectrum [28] in the limit that the specific heat ratio approaches unity. To leading order in dispersion this term causes a shift of $\left(2 \Gamma^{2} k^{3} / c\right)$ to the right in the mean frequency of the line.

The third term of eq (136) is also antisymmetric about $\omega=-c k$. It has the effect of shifting the mean frequency to the right by $(5 b \Gamma k / a)$. This represents a very small shift, which by (137) is less than that of the previous dispersion term which is itself usually neglected in practical applications.

Upon comparison with the undamped wave solution, the fourth term of eq (136) is seen to originate from the decreasing wave speed or increasing inertia in the rightward direction. This term is symmetric about $\omega$ $=-c k$ and, to leading order, increases the width of the line by $3 b c / 4 a$. An increase in width corresponds physically to a larger effective attenuation coefficient.

The fifth term of eq (136) is symmetric about $\omega=-c k$ and, by the definition (139), results in a decrease in width of $3 b c / 4 a$. Thus the fourth and fifth terms effectively, if not identically, cancel each other. One possible physical interpretation of the latter term is as follows. The Fourier component of wavenumber $k$ of the rightward pulse has a lifetime of $\left(\Gamma k^{2}\right)^{-1}$, and therefore a mean path length $\mathrm{c} / \Gamma k^{2}$. Equations (115-117) imply a distance-dependent attenuation factor

$$
\Gamma(x)=\Gamma\left(1-\frac{b x}{a}\right)
$$


from which we infer that the effective attenuation coefficient of the kth Fourier component of the rightward pulse is

$$
\Gamma_{\text {eff }}=\Gamma-f_{1} \frac{b c}{a k^{2}}
$$

where $f_{1}$ is a positive number of order unity, and the resulting width of the Brillouin line is $\Gamma_{\text {eff }} k^{2}$. The full analysis then gives $f_{1}=3 / 4$.

Hence the near-cancellation of the previous two terms may be an artifact of setting $\beta=0$ in eq (117). For a more general solution where, for example, wave speed and damping increase in opposite directions, the corresponding new terms might reinforce each other. In any case, measurement of such a width change would be difficult as it would require a fractional precision in the width measurement equal to the fractional change in attenuation over the path length of the monitored Fourier component.

Finally, the last term in eq (136) is highly oscillatory and qualitatively similar to the third derivative of the Lorentzian. Like its counterpart in the diffusion solution, it would be very difficult to detect and may be neglected.

Similar observations, with appropriate changes of sign in some cases, apply to $S_{\ell}(k, \omega)$, the anti-Stokes line centered at $\omega=c k$ resulting from the leftward pulse.

\section{Summary}

As an initial step toward the understanding of Rayleigh-Brillouin scattering in a temperature gradient, which involves nonuniform, three-dimensional coupled damped wave and diffusion equations, we have analyzed in detail the initial value problem for a set of prototype one-dimensional differential equations with linearly nonuniform coefficients, to first order in nonuniformity.

A question which first arose was whether explicit boundaries were necessary for the system, since at large distances the differential equations become unphysical. For the models studied, it is possible to derive meaningful solutions of the initial value problem without such boundaries for sufficiently narrow initial pulses and sufficiently short times. Also, if the equation has a damping mechanism, it is possible to construct a meaningful dynamic structure factor for values of $k$ whose Fourier components are damped sufficiently rapidly. Conditions for the validity of solutions are listed in table 1 .

Our procedure has been first to Fourier transform in space and Laplace transform in time. The resulting equations, algebraic in the uniform case, become first-order differential equations in $k$ for the linear nonuniform case. An expansion through quadratic order in nonuniformity would lead to a second-order differential equation in $k$, for which there is no general method of solution.

We have seen that the condition of small nonuniformity cannot be represented by any dimensionless parameter in $F$-space or $F L$-space. Therefore, to construct the time evolution of the pulse and, ultimately, the dynamic structure factor, we must follow the circuitous path indicated in figure 1 . The ability to perform the initial inverse Fourier transform restricts the range of solvability of the linear differential equation in $k$. One of our initial goals, the solution of the nonuniform damped wave equation with arbitrary, independent variation of the nonuniformity in both wave speed and damping, for this reason has not been realized.

We have derived explicit solutions to the initial value problem for the nonuniform undamped wave, diffusion, and (for a special case) damped wave equations. These solutions display properties expected on intuitive grounds, and reduce properly to their respective uniform solutions.

Throughout the derivations, certain mathematical liberties have been taken. We have assigned small imaginary parts to intrinsically real coefficients, although these imaginary components go to zero at the end of the calculation. For the diffusion and damped wave equations, we have altered the measure of integration 
in the final solution to suppress singularities at large distance. In the damped wave equation, we have introduced and postulated properties of an operator which simultaneously performs an inverse Laplace transformation and projects out the leading term in a dispersion expansion. Attempts to justify these liberties have been based on pragmatism and physical intuition rather than mathematical rigor, although we conjecture that such steps could (probably) be justified mathematically. Of course, an a posteriori justification is that our solutions explicitly satisfy the differential equations through linear order in nonuniformity, and that our initial conditions specify a unique solution.

Since the present formalism neglects coupling between wave and diffusion modes, three-dimensional effects, finite initial time derivatives and position-dependent rates of generation of fluctuations, one should be cautious in drawing conclusions from these results about experimental Rayleigh-Brillouin scattering from a fluid in a temperature gradient. However, the results suggest that further studies of possible changes in the Rayleigh line could be fruitful. Kirkpatrick, et al. [6] have noted that certain modifications of the Brillouin spectrum vanish when an average over a finite volume of the fluid is taken. But according to the simple and intuitive arguments of section 5.2 , all heat modes within a given volume should experience a drift velocity in the direction of increasing thermal diffusivity. Our one-dimensional results suggest that the Rayleigh line is thus Doppler shifted. Detection of such a shift, if it persists in the coupled threedimensional problem, depends on precision calibration of the incoming laser frequency and precision measurement of the mean frequency of the Rayleigh line. The shift would be most pronounced for systems whose thermal conductivity changes rapidly with temperature and for large values of $k$. By contrast, the asymmetry in the size of the Brillouin peaks predicted by previous authors, [1-10] and not considered here, is generally agreed to be inversely proportional to $k^{2}$, and experiments to detect such an effect [14] have thus been performed at very small values of $k$.

The author thanks R. D. Mountain for elucidating many aspects of light scattering theory and for guidance and encouragement. He thanks H. J. Raveche and the staff of the Thermophysics Division, National Bureau of Standards, Washington, DC for their hospitality during the author's visit in 1979, when much of this work was completed. He also thanks S. W. Haan, R. J. Rubin and J. R. Dorfman for valuable suggestions.

\section{References}

According to conversations with J. R. Dorfman and G. van der Zwan, the authors of refs. [1-10] have now formed a consensus that the correct leading-order spectrum is that given in refs. $[6,9$, and 10$]$.

[1] Procaccia, I.; Ronis, D.; Oppenheim, I. Light scattering from nonequilibrium states: the implication of broken time-reversal symmetry. Phys. Rev. Lett. 42(5): 287-291, 1979 January 29.

[2] Procaccia, I.; Ronis, D.; Collins, M. A.; Ross, J.; Oppenheim, I. Statistical mechanics of stationary states. I. Formal theory. Phys. Rev. A 19(3): 1290-1306; 1979 March.

[3] Ronis, D.; Procaccia, I.; Oppenheim, I. Statistical mechanics of stationary states. II. Application to low-density systems. Phys. Rev. A 19(3): 1307-1323; 1979 March.

[4] Ronis, D.; Procaccia, I.; Oppenheim, I. Statistical mechanics of stationary states. III. Fluctuations in dense fluids with applications to light scattering. Phys. Rev. A 19(3): 1324-1339; 1979 March.

[5] Kirkpatrick, T.; Cohen, E. G. D.; Dorfman, J. R. Kinetic theory of light scattering from a fluid not in equilibrium. Phys. Rev. Lett. 42(14). 862-865; 1979 April 2.

[6] Kirkpatrick, T.; Cohen, E. G. D.; Dorfman, J. R. Hydrodynamic theory of light scattering from a fluid in a nonequilibrium steady state. Phys. Rev. Lett. 44(7): 472-475; 1980 February 18.

[7] Van der Zwan, G.; Mazur, P. Light scattering from a fluid with a stationary temperature gradient. Phys. Lett. 75A(5): 370-371; 1980 February 4.

[8] Van der Zwan, G. On some aspects of fluctuating hydrodynamics. Thesis. Ryksuniversiteit te Leiden; 1980.

[9] Tremblay, A.-M. S.; Siggia, E. D.; Arai, M. R. Fluctuations about hydrodynamic nonequilibrium steady states. Phys. Lett. 76A(1): $57-60 ; 1980$ March 3.

[10] Ronis, D.; Putterman, S. Simplified hydrodynamic therory of nonlocal stationary state fluctuations. Phys. Rev. A 22: 773-777; 1980 August.

[11] Mountain, R. D. Spectral distribution of scattered light in a simple fluid. Rev. Mod. Phys. 38(1): 205-214; I966 January.

[12] Komarov, L. I.; Fisher, I. Z. Theory of Rayleigh scattering of light in liquids. Soviet Phys. J.E.T.P. 16(5): 1358-1361; 1963 May.

[13] Pecora, R. Doppler shifts in light scattering from pure liquids and polymer solutions. J. Chem. Phys. 40(6): 1604-1614; 1964 March 15. 
[14] Beysens, D.; Garrabos, Y.; Zalczer, G. Experimental evidence for Brillouin asymmetry induced by a temperature gradient. Phys. Rev. Lett. 45(6): 403-406; 1980 August 11.

[15] Brownell, J. H. Wave number space analysis of propagation in nonuniform media. Am. J. Phys. 41(2): 207-212; 1973 February.

[16] Clark, N. A. Inelastic light scattering from density fluctuations in dilute gases. The kinetic-hydrodynamic transition in a monatomic gas. Phys. Rev. A 12(1): 232-244; 1975 July.

[17] Landau, L.; Placzek, G. Structure of the undisplaced scattering line. Physik Z. Sowjetunion 5: $172 ; 1934$.

[18] Reif, F. Statistical and thermal physics. New York: McGraw-Hill; 1965; p. 301.

[19] Mountain, R. D. Generalized hydrodynamics. Adv. Mol. Relax. Proc. 9: 225-291; 1976.

[20] Mathews, J.; Walker, R. L. Mathematical methods of physics. New York: Benjamin; 1965; p. 3.

[21] Dym, H.; McKean, H. P. Fourier series and integrals. New York: Academic Press; 1972; p. 116.

[22] Gradshteyn, I. S.; Ryzhik, I. M. Tables of integrals, series and products. New York: Academic Press; $1965 ;$ p. 307.

[23] Richardson, L. F. Atmospheric diffusion shown on a distance-neighbour graph, Proc. Roy. Soc. London Series A 110(756): 709-737; 1926 April.

[24] Berg, P. W.; McGregor, J. L. chapter 3 in Elementary partial differential equations. San Francisco: Holden-Day; 1966; 29-33.

[25] McQuarrie, D. A. Statistical mechanics. New York: Harper and Row; 1976; p. 563.

[26] Crandall, I. B. Theory of vibrating systems and sound. New York: Van Nostrand; 1926; p. 110.

[27] Berg, P. W.; McGregor, J. L. chapter 8 in Elementary partial differential equations. San Francisco: Holden-Day; $1966 ;$ p. $181-187$.

[28] Mountain, R. D. Liquids: dynamics of liquid structure. Chem. Rubber Co. Crit. Revs. Solid State 1(1): 5-46; 1970. 